\title{
Sex Differences in the Response to Different Tinnitus Treatment
}

\section{OPEN ACCESS}

Edited by:

Christopher R. Cederroth, Karolinska Institutet (KI), Sweden

Reviewed by: Eleftheria Vellidou,

Institute of Communication and Computer Systems, Greece Myra Spiliopoulou,

Otto von Guericke University

Magdeburg, Germany

${ }^{*}$ Correspondence:

Sarah Michiels

sarah.michiels@uantwerpen.be Annick Gilles

annick.gilles@uza.be

tThese authors have contributed equally to this work

Specialty section:

This article was submitted to Auditory Cognitive Neuroscience, a section of the journal

Frontiers in Neuroscience

Received: 14 January 2020 Accepted: 07 April 2020

Published: 12 May 2020

Citation:

Van der Wal A, Luyten T,

Cardon E, Jacquemin L,

Vanderveken OM, Topsakal V, Van de Heyning P, De Hertogh W, Van

Looveren N, Van Rompaey $V$, Michiels S and Gilles A (2020) Sex

Differences in the Response

to Different Tinnitus Treatment.

Front. Neurosci. 14:422

doi: 10.3389/fnins.2020.00422
Annemarie Van der Wal1,2t, Tine Luyten ${ }^{1,3+}$, Emilie Cardon ${ }^{1,3+}$, Laure Jacquemin ${ }^{1,3}$, Olivier M. Vanderveken 1,3, Vedat Topsakal1,3, Paul Van de Heyning 1,3, Willem De Hertogh ${ }^{2}$, Nancy Van Looveren ${ }^{4}$, Vincent Van Rompaey ${ }^{1,3}$, Sarah Michiels ${ }^{1,2,3 *}$ and Annick Gilles ${ }^{1,3,5 *}$

${ }^{1}$ Department of Otorhinolaryngology - Head and Neck Surgery, Antwerp University Hospital, Edegem, Belgium, ${ }^{2}$ Department of Rehabilitation Sciences and Physiotherapy, Antwerp University, Antwerp, Belgium, ${ }^{3}$ Department of Translational Neurosciences, Antwerp University, Antwerp, Belgium, ${ }^{4}$ Hoorzorg Van Looveren BVBA, Borsbeek, Belgium, ${ }^{5}$ Department of Education, Health and Social Work, University College Ghent, Ghent, Belgium

Introduction: Tinnitus is a complex symptom requiring a thorough multidisciplinary assessment to construct an individual's tinnitus profile. The Antwerp University Hospital hosts a tertiary tinnitus clinic providing intensive, multidisciplinary tinnitus care in the form of combinational psychological treatment with either Tinnitus Retraining Therapy (TRT)/Cognitive Behavioral Therapy (CBT) or TRT/eye movement desensitization and reprocessing therapy (EMDR), high-definition transcranial direct current stimulation (HDtDCS), and physical therapy treatment (in cases of somatic influence of the neck or the temporomandibular area). Several factors may contribute to therapy effect of which the role of gender has recently gained more interest. As such, the current manuscript explores gender differences in the outcome of different tinnitus treatments.

Methods: Data on treatment outcome of four distinct tinnitus treatments (1. HDtDCS; 2. orofacial physical therapy; 3. combination TRT + CBT; and 4. combination TRT + EMDR) were pooled and compared. Treatment outcome was assessed via the Tinnitus Functional Index (TFI). Participants completed the TFI at baseline, immediately after treatment and after 9 weeks ( \pm 3 weeks) follow-up. To explore the effect of gender on different treatment outcomes, a linear mixed model was designed including Time point, Gender, and Therapy Group as fixed factors as well as all interactions between these factors.

Results: TFI scores improved significantly over time regardless of therapy group $(p<0.0001)$. A mean TFI decrease of at least 13 points was obtained by all participants except by those in the HD-tDCS. Significant interactions between Gender and Time point were identified in all groups except for the TRT +EMDR group. Female subjects improved more extensively than males in the HD-tDCS $(p=0.0009)$ and orofacial therapy group $(p=0.0299)$. Contrarily, in the TRT +CBT group, male participants showed a significant improvement whereas the mean TFI scores of female subjects remained on baseline levels $(p=0.0138)$. 
Conclusion: Our data suggest that male and female tinnitus patients seem to react differently to different therapy options. We strongly encourage further prospective studies to discern the relevance of gender in therapy outcome.

Keywords: gender, tinnitus, gender-related outcome, TRT, CBT, neuromodulation, HDtDCS, tDCS

\section{INTRODUCTION}

Tinnitus, the perception of sound in the absence of an external sound source, is a frequently experienced symptom in modern society. The prevalence of tinnitus in an adult population is around 15\% (Seidman and Jacobson, 1996; Gilles et al., 2012). In $2-3 \%$ of patients, the tinnitus is sufficiently bothersome to affect the quality of life due to the association with anxiety, depression, sleep disorders, concentration difficulties, and elevated stress levels (Henry et al., 2005). As a consequence, many patients find their way to the clinic seeking for alleviating treatment. In the management of tinnitus, a multi-disciplinary approach is essential in order to tailor therapy toward the patient's requirements and needs. Thorough evaluation of the patient comprises a systematic history including tinnitus characteristics, potential tinnitus triggers, and the evaluation of coexisting symptoms such as subjective hearing loss, otalgia, decreased speech understanding (Gilles et al., 2016), vertigo, and hyperacusis (Van de Heyning et al., 2015). The presence of cervical spine dysfunction as well as bruxism or known history of temporomandibular dysfunctions should be inquired and assessed accordingly as somatosensory influences may cause tinnitus and/or increase pre-existing tinnitus loudness or alter pitch (Michiels et al., 2015a, 2018b). In addition, the comorbidity with depression or anxiety disorders should be assessed, as emotional factors typical in these disorders are strong predictors of poor adjustment to the symptom of tinnitus (Zoger et al., 2006; Pattyn et al., 2016). Following extensive anamnesis, further multidisciplinary investigations as well as imaging may be required leading toward an individual tinnitus profile guiding the patient toward the most appropriate patient-specific therapy (Van de Heyning et al., 2015).

Yearly, approximately 1500 patients consult the tertiary expertise tinnitus clinic [Tinnitus Treatment and Research Centre Antwerp (TINTRA)] at the Antwerp University Hospital, with tinnitus being their primary complaint. Through a multidisciplinary approach, patients receive treatment according to their tinnitus profile, underlying causes/mediators and psychological burden. For all patients, pre-therapeutic tinnitus burden is measured at baseline, post-therapy, and at 9 weeks ( \pm 3 weeks) follow-up moment using tinnitus questionnaires.

Several treatments provided at TINTRA are psychology-based focusing on altering the coping strategies in order to change emotional responses toward the tinnitus. Cognitive Behavioral Therapy (CBT) in particular aims to change non-constructive cognitive distortions/behaviors and develop personal coping strategies targeting the tinnitus issues. Tinnitus Retraining Therapy (TRT) on the other hand is a habituation therapy in which directive counseling aims to reclassify the tinnitus percept to a neutral signal in combination with the use of sound therapy. Both CBT and TRT have proven to be effective in the treatment of tinnitus (Jastreboff and Jastreboff, 2000; Jastreboff, 2007, 2015; Cima et al., 2014). Eye movement desensitization and reprocessing (EMDR) therapy is a form of psychotherapy in which the patient is enquired to recall distressing thoughts/images (i.e., associated with the tinnitus) after which the therapist directs the patient with bilateral sensory input (i.e., hand tapping, auditory stimuli, or sideto-side eye movements). EMDR is a widely used technique in the treatment of post-traumatic stress disorder (Guideline Development Panel for the Treatment of PTSD in Adults, and American Psychological Association, 2019). In the field of tinnitus, a first preliminary study on EMDR was recently published showing promising results as significant improvement on tinnitus burden (measured by the Tinnitus Handicap Inventory) and depressive symptoms (measured by the Beck Depression Inventory) was shown up until 6 months after EMDR treatment (Phillips et al., 2019).

Also, transcranial direct current stimulation (tDCS) has been a topic of research at TINTRA. TDCS is a form of neuromodulation delivering a constant, low direct current to the brain through electrodes positioned on the head. Up until now, a total of 31 studies evaluated the effects of tDCS on tinnitus reporting various degrees of effect, ranging from no effect to significant tinnitus reduction. A large heterogeneity in used tDCS protocols and outcomes is apparent which constrains the comparability of these studies (Lefaucheur, 2016; Lefaucheur et al., 2017). At our department tDCS trials have shown clinically significant improvement in $32 \%$ of patients with large interindividual variability (Jacquemin et al., 2018).

For patients who experience a somatosensory influence on tinnitus originating in a dysfunction of the cervical spine or temporomandibular area (Michiels et al., 2015b, 2016b, 2018a), physiotherapy treatment is provided. Depending on the area of the dysfunction that is primarily influencing the tinnitus, the physical therapy treatment is adjusted. In case of a primary influence from the cervical spine, a multimodal manual physical therapy can be provided (Michiels et al., 2016a, 2017). This type of treatment showed a significant improvement on the global perceived effect in $53 \%$ of the patients in our clinic (Michiels et al., 2016c). In case temporomandibular dysfunctions are primarily influencing the tinnitus, orofacial physiotherapy is provided, when needed combined with occlusal splints provided by the dentist. A recent systematic review on the topic showed promising results, although differences in treatment modalities and outcome measures make it hard to draw any definitive conclusions (Michiels et al., 2019).

As the tinnitus population is highly heterogeneous, a onetherapy-fits-all approach is non-existent and many treatments may add to tinnitus alleviation depending on the patients' 
tinnitus profile. Due to the heterogeneity, treatment outcomes also tend to vary tremendously. Recently the role of gender on the perception of tinnitus has gathered more attention. In a study by Seydel et al. (2013), women showed more tinnitus-related distress compared to men with this effect depending on age and duration of the tinnitus. These results were partially confirmed by a recent study by Han et al. (2019) and some authors have mentioned gender difference in the amount of tinnitus-related distress as well (Hiller and Goebel, 2006), whereas others did not find any gender differences (Meric et al., 1998; Pinto et al., 2010). As a result, the role of gender remains elusive. Recently, severe tinnitus was shown to be associated with an increased risk of suicide attempts in female patients only (Lugo et al., 2019). Interestingly, those patients who had been diagnosed with tinnitus in a clinical setting were no longer at risk, highlighting the need for specialty care in the tinnitus population. If males and females perceive tinnitus differently and, as a consequence, possibly show distinct reactions toward therapeutic intervention, caregivers should take these gender differences into account. The current manuscript reports on the effects of gender on the outcomes of the distinct tinnitus therapies provided at TINTRA.

\section{MATERIALS AND METHODS}

\section{Study Protocol}

The current manuscript describes the effects of gender on tinnitus treatment outcome. Therefore, all data collected prior to treatment, post-treatment, and at a follow-up visit ( $9 \pm 3$ weeks) of patients who received tinnitus treatment, were analyzed retrospectively. All patients had chronic, non-pulsatile, subjective tinnitus for longer than 3 months. Patients with active middle ear pathology were excluded from analysis. Pure tone audiometry was performed at baseline using a two-channel Interacoustics AC-40 audiometer and headphones in a soundproof booth. Air conduction thresholds were measured at 125, 250, 500, 1000, 2000, 3000, 4000, 6000, and $8000 \mathrm{~Hz}$. Bone conduction thresholds were tested at 250, 500, 1000, 2000, 3000, and $4000 \mathrm{~Hz}$. Tinnitus evaluation was performed using selfadministering questionnaires.

\section{Treatment}

\section{Psychological Treatment}

Two combination psychological therapies were provided with either TRT +CBT combination (five sessions TRT/five sessions $\mathrm{CBT}$ ) or TRT+EMDR combination (five sessions TRT/five sessions EDMR). All therapies were provided by licensed TRT audiologists and CBT/EMDR psychologists. The full approach of this bimodal psychotherapy is described in Luyten et al. (2019).

\section{High-Definition Transcranial Direct Current Stimulation}

Patients received six sessions of high-definition tDCS (HD-tDCS) over 3 weeks' time (2x/week) with silver/silverchloride electrodes placed on the right dorsolateral prefrontal cortex (dLPFC). A direct current of $2 \mathrm{~mA}$ was applied with a $20 \mathrm{~s}$ fade-in/fadeout time delivered by a battery-driven $1 \times 1$ tDCS low-intensity stimulator and $4 \times 1$ multichannel stimulation adaptor (Soterix Medical Inc., New York, NY, United States) as described in the HD-tDCS stimulation guidelines (Villamar et al., 2013). During each session, the patient received 20 min of stimulation.

\section{Conservative Temporomandibular Treatment}

Patients received a maximum of 18 sessions of orofacial therapy during a fixed time window of 9 weeks. This therapy was primarily directed to the temporomandibular joint and masticatory muscles. If present, cervical spine dysfunctions were treated as well, using a combination of manual mobilizations and exercise therapy. In case of severe bruxism, an occlusal splint was provided in addition to the physiotherapy. Therapists, providing the orofacial physiotherapy, were trained to the study protocol prior to the start of the study. More details can be found in the published study protocol (Michiels et al., 2018b).

\section{Treatment Allocation}

Treatment allocation to one of the four interventions described below was based on patients' complaints and needs as discussed during an intake visit at the TINTRA tinnitus consultation. For instance, only patients with self-reported temporomandibular complaints were considered for the orofacial therapy. Different therapy options were discussed based on individual patients' tinnitus profiles, and final treatment decisions were made by the patients. Thus, a certain level of selection bias was present in the treatment allocation, but this was solely based on individual patients' needs. Demographic variables, such as gender, were never taken into account as a deciding factor for treatment choice.

\section{Ethics}

All patients filled out an informed consent in which permission was granted to use their data gathered during and prior to tinnitus treatment. Ethics Committee approval numbers involved in the current analysis are 16/41/415, 16/35/360, and 16/48/513.

\section{Questionnaires}

\section{Tinnitus Functional Index}

The Tinnitus Functional Index (TFI) (Meikle et al., 2012; Rabau et al., 2014) consists of 25 items assessing the tinnitus severity as well as the impact of tinnitus in daily life. In addition to a total score reflecting the total tinnitus burden, eight subscales define the level of inconvenience for the following aspects: intrusiveness, reduced sense of control, cognitive interference, sleep disturbance, auditory difficulties, interference with relaxation, reduced quality of life, and emotional distress. The TFI has proven to be useful in the assessment of treatmentrelated changes in tinnitus. As such, a reduction of 13 points on the total TFI score after tinnitus treatment is considered as a clinically relevant and subjectively perceived reduction for the patient (Meikle et al., 2012).

\section{Visual Analog Scale}

The mean tinnitus loudness throughout the day was assessed by use of a Visual Analog Scale (VAS). In this case, the patient had to indicate the mean tinnitus loudness over the last week on a scale 
from 0 (no tinnitus at all) to 100 (the most extreme loudness one can imagine) by use of a ruler.

\section{Hyperacusis Questionnaire}

The Hyperacusis Questionnaire (HQ) (Khalfa et al., 2002; Meeus et al., 2010) determines the presence/absence of hyperacusis. According to Khalfa's original HQ, one can speak of hyperacusis when the score on the HQ is 28 or higher (Khalfa et al., 2002).

\section{Hospital Anxiety and Depression Scale}

The Hospital Anxiety and Depression Scale (HADS) is a screening tool to detect symptoms of anxiety and/or depression (Zigmond and Snaith, 1983). The HADS consists of 14 items in total assessing increased signs of anxiety (seven items) or depression (seven items). For each of the subscales, a score between 8 and 10 is considered as "borderline" while a score of 11 and higher is considered as "case."

\section{Statistics}

A linear mixed model was designed using $\mathrm{JMP}^{\circledR}$ Pro software $\left(\mathrm{JMP}^{\circledR}\right.$ Pro, Version 14.0.0, 2018 SAS Institute Inc., Cary, NC, United States, 1989-2019) to explore the effects of gender on outcomes of different tinnitus treatments. Total TFI score was chosen as the outcome variable. The model applied the following fixed factors: Time point (baseline, post-treatment, follow-up), Gender (male, female), and Therapy group (HD-tDCS, orofacial therapy, TRT combined with CBT, TRT combined with EMDR). All possible two-way interactions between these factors were added to the model, as well as the three-way interaction Time point* Gender*Therapy group. Possible confounding factors were added to the model in a stepwise additive manner. These factors were age, hearing level (pure tone averages for 1, 2, and $4 \mathrm{kHz}$ ), tinnitus characteristics (type, duration, and laterality of the tinnitus), scores on the HADS depression and anxiety subscales, and scores on the HQ. Considerably more men than women were in all therapy groups except for the orofacial therapy group, in which the gender distribution was more balanced. Participant was added as a random intercept. Post hoc analyses were performed using linear mixed models for each of the four therapy groups separately. Here, Time point and Gender were added as fixed factors with an additional two-way interaction of Time point* Gender. $P$-values of $<0.05$ were considered significant.

\section{RESULTS}

Data from 316 patients were included in the analysis. An overview of patients' characteristics at baseline can be found in Table 1. Considerably more men than women were included in all therapy groups except for the orofacial therapy group, in which the gender distribution was more balanced. Age varied slightly between therapy groups, with mean age in the HDtDCS group being the highest and participants in the orofacial therapy group being the youngest. Hearing levels did not differ significantly between groups, nor did tinnitus characteristics (duration, type, and laterality of the tinnitus) or TFI scores at baseline. Some group level differences were found for the

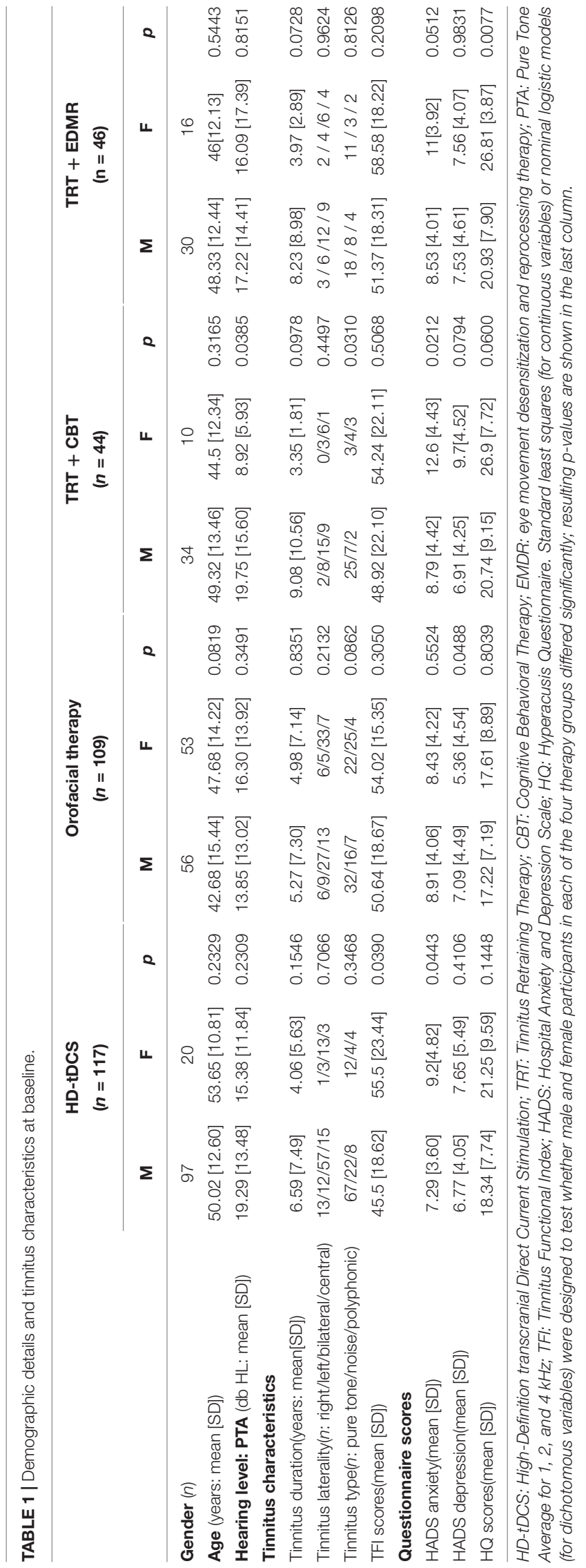


TABLE 2 | Results of the linear mixed model analysis.

p-value

\section{Fixed factors}

Time point

Gender

Therapy group

HADS depression subscale scores

$\mathrm{HQ}$ scores

\section{Interactions}

Time point * Gender

HADS: Hospital Anxiety and Depression Scale; HQ: Hyperacusis Questionnaire.

additional questionnaire scores, with participants in the HDtDCS and orofacial therapy groups scoring lower on the anxiety subscale of the HADS and on the HQ than patients in both psychotherapeutic groups.

Results of the linear mixed model analysis are summarized in Table 2. Of all putative confounding factors, only the HADS depression subscale score and the HQ score were found to contribute significantly to the model. All other possible confounding factors (age, hearing level, tinnitus characteristics, and HADS anxiety subscale scores) did not have any demonstrable effect on the model and were excluded from the analysis. Thus, the final model included two additional factors (HADS depression scores and HQ scores) next to the fixed factors Time point, Gender, and Therapy group. Overall, a significant fixed effect of time point was found, with TFI scores decreasing over time for all therapy groups and for both genders $(p<0.0001)$ (Figure 1A). Additionally, the main effect of gender was found to be significant, with women generally having a higher TFI score than men $(p=0.0496)$ (Figure 1B). A significant interaction between Time point and Therapy group was identified $(p=0.0023)$, indicating that TFI scores of participants in different therapy groups evolved differently over time. Crucially, a significant three-way interaction between Time point, Gender, and Therapy group was found $(p=0.0002)$. This interaction implies that treatment response in the different therapy groups was modulated by gender (Figure 2).

To further explore this highly significant three-way interaction, post hoc analyses were performed for each therapy group. Similar to the primary analysis, HADS depression subscale scores and HQ scores were added to the model as confounding factors. In three out of four therapy groups, significant interactions between Time point and Gender were found. These interactions indicate that the evolution of TFI scores depended on gender in these specific therapy groups. In the HD-tDCS (Time point*Gender: $p=0.0009$ ) and orofacial therapy group (Time point*Gender: $p=0.0299$ ), female participants generally had higher TFI scores at baseline, but improved more extensively than male subjects (Figures 2A,B). On average, TFI scores of female participants in the HD-tDCS group decreased by $15.83 \pm 24.27$ points from baseline to follow-up, whereas
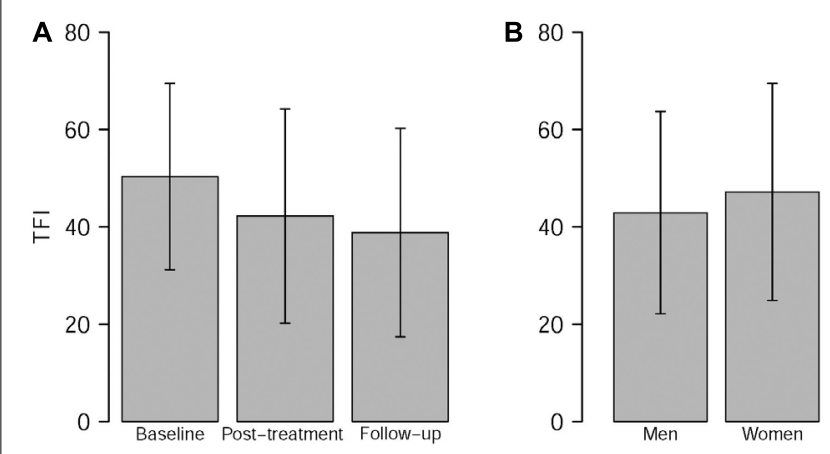

FIGURE 1 | Main effects of time point (A) and gender (B). (A) TFI scores change significantly over time, independently of therapy group or gender ( $p<0.0001)$. TFI scores at baseline: $50.34 \pm 19.13$; TFI scores at the post-treatment time point: $42.24 \pm 21.99$; TFI scores at follow-up: $38.84 \pm 21.39$; mean \pm SD. (B) TFI scores are higher in women than in men, independently of therapy group or time point $(p=0.0496)$. TFI scores for women: $47.19 \pm 22.28$; TFI scores for men: $42.93 \pm 20.79$; mean $\pm S D$. Error bars represent SD. TFI: Tinnitus Functional Index.

male participants' TFI scores dropped by only $2.53 \pm 13.79$ points. In the orofacial therapy group, female participants' TFI scores decreased by $19.02 \pm 16.85$ points while scores of male participants decreased by $10.53 \pm 19.65$ points.

Contrarily, in the TRT + CBT group, male subjects improved significantly over time whereas female participants' mean TFI scores only decreased slightly after treatment $(p=0.327)$ but did not remain stable at follow-up (Figure 2C) (Time point*Gender: $p=0.0138$ ). From baseline to follow-up, male participants' TFI scores decreased by $22.42 \pm 23.02$ points whereas TFI scores of female subjects in this group actually increased marginally with $0.44 \pm 21.26$ points. Finally, in the TRT + EMDR group, the two-way interaction Time point* Gender was not significant $(p=0.5240)$, with men and women improving over time in a highly similar way (Figure 2D). The decrease in TFI scores from baseline to follow-up was $19.1 \pm 18.52$ and $16.46 \pm 14.3$ points in female and male participants, respectively. 
A

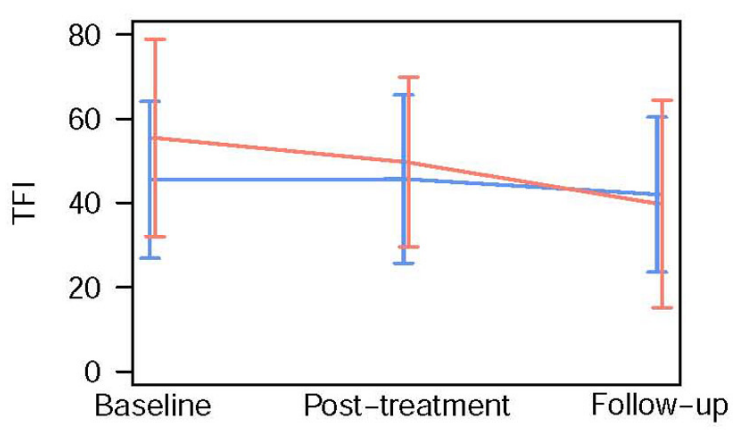

C

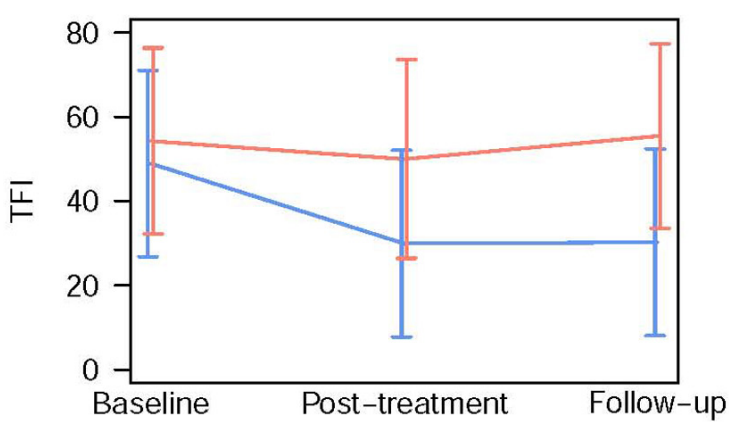

B

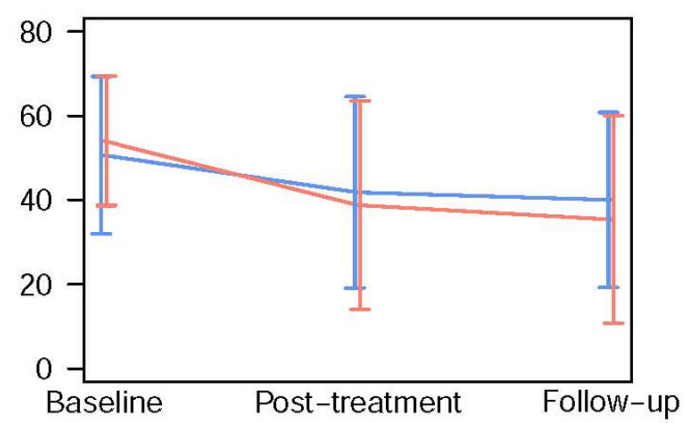

D

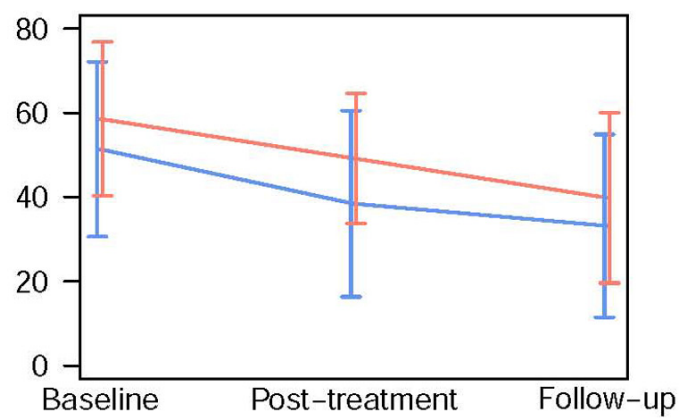

FIGURE 2 | Treatment outcome in different therapy groups differs according to gender. Blue lines represent male subjects and red lines represent female subjects. Data are presented as mean TFI scores for each time point \pm SD. (A) Female participants benefit more from HDtDCS treatment than men. TFI scores decreased more in female participants $(-15.83 \pm 24.27)$ than male $(-2.53 \pm 13.79)$ from baseline to follow-up. (B) Female participants benefit more from orofacial therapy than men. TFI scores decreased more in female participants $(-19.02 \pm 16.85)$ than male $(-10.53 \pm 19.65)$ from baseline to follow-up. (C) Male participants benefit more from TRT combined with CBT than women. TFI scores decreased more in male participants $(-22.42 \pm 23.02)$ than female $(0.44 \pm 21.26)$ from baseline to follow-up. (D) Male and female participants benefit equally from TRT combined with EMDR. TFI scores decreased similarly in male $(-19.1 \pm 15.82)$ and female participants (-16.46 \pm 14.3$)$ from baseline to follow-up. TFI: Tinnitus Functional Index; HDtDCS: High-Definition transcranial Direct Current Stimulation; TRT: Tinnitus Retraining Therapy; CBT: Cognitive Behavioral Therapy; EMDR: Eye Movement Desensitization and Reprocessing.

HADS depression subscale scores and HQ scores were found to contribute significantly to the model. Post hoc analyses revealed that both questionnaire scores had a significant effect on tinnitus severity, with higher scores on both questionnaires equaling higher total TFI scores. These effects were not dependent on either Time point or Gender, as evidenced by the non-significance of the interactions between these questionnaire scores and Time point ( $p=0.5914$ for HADS depression scores, $p=0.1814$ for HQ scores) or Gender ( $p=0.6806$ for HADS depression scores, $p=0.1868$ for HQ scores).

\section{DISCUSSION}

The large variability in tinnitus treatment outcomes may be driven by differences in individual patient characteristics. The current manuscript retrospectively explored whether gender may account for any of this heterogeneity. We report remarkable gender effects on treatment outcomes of several tinnitus therapy options. Overall, our results indicate that gender might be an influential mediator of treatment outcome.

In a large group of tinnitus patients, we found that women benefited from orofacial physiotherapy to a greater extent than men. In the general population, a recent meta-analysis showed that the risk for developing temporomandibular disorders (TMD) is twice as high in women than in men (Bueno et al., 2018). Likewise, a study of Vielsmeier et al. (2011) showed that significantly more women than men had TMD in a population of patients with tinnitus, something which was also found in the general population (Edvall et al., 2019). Therefore, we hypothesize that the proportion of true TMD sufferers was higher in female than in male patients. This gender difference in baseline TMD burden may then explain the higher success ratio of conservative orofacial treatment in female tinnitus patients. Currently, no other studies reported on gender differences in orofacial treatment effect on tinnitus complaints. It must also be noted that although statistically significant, the difference in decrease in TFI score between males and females in this treatment group is small. Our results should therefore be 
confirmed in future research, specifically designed to investigate gender differences.

In our study population, women also demonstrated a better treatment response after receiving consecutive sessions of HDtDCS. These results are in agreement with Frank et al. (2012), who reported larger beneficial effects of frontal tDCS in women. Both protocols comprised anodal stimulation of the right dLPFC, suggesting that this gender difference might be driven by true underlying physiological differences. Indeed, dLPFC volume has been shown to be greater in women than men (Schlaepfer et al., 1995; Luders et al., 2009), and anodal tDCS of the prefrontal cortex might have greater effects in females (Fumagalli et al., 2010). These findings cannot be explained by the amount of current intensity going through the brain as it was previously shown that females receive significantly less current compared to males when targeting parietal and frontal areas due to more dense parietal bone in females (Russell et al., 2014). A significant difference in TFI at baseline between males and females was observed with females showing higher TFI scores which may imply a greater opportunity for improvement in this population. However, even after correcting for this difference in baseline TFI score, the effect of gender over time remained significant. In addition, no equal proportion of male and female subjects was obtained in this therapy group so these results need to be interpreted with caution.

Conversely, in our study population, male patients benefited more than females from TRT combined with CBT, while both males and females experienced significant improvement through EMDR therapy. Although some evidence exists for gender differences in tinnitus perception and distress, literature on gender effects on the efficacy of psychological tinnitus treatments is scarce. To date, meta-analyses on psychotherapy outcomes report minimal to no gender differences (Bowman et al., 2001; Felmingham and Bryant, 2012; Swift et al., 2013; Tao et al., 2015; Budge and Moradi, 2019).

In a study reporting on the long-term effects of TRT combined with cognitive-behavioral elements, Seydel et al. (2010) reported subtle gender differences on treatment outcome. Male and female patients showed similar outcomes of this treatment overall, but women experiencing longer ( $>2$ years and especially $>10$ years) tinnitus duration were less likely to maintain positive treatment effect at a 1-year follow-up time point. In our dataset, we did not identify any effects of tinnitus duration. However, the relatively long average tinnitus duration (7.78 years) might account for this gender difference on the outcome of a treatment combining TRT and CBT. About 70\% of the female participants included in the TRT + CBT group reported a psychiatric diagnosis in the present or the past. These findings suggest that 10 sessions might not be sufficient to alleviate the complex tangle of aggravating complaints. The proportion of patients with psychiatric comorbidity was lower in the TRT + EMDR group. Given the fact that $40 \%$ of female participants did report a significant decrease of 13 points or more in the TRT + CBT group, we emphasize the identifiable influence of psychiatric comorbidity on treatment outcome. However, the cognitive behavioral approach could have influenced therapy outcome on the basis of the cognitive therapeutic techniques that were used. Some evidence can be found on women to experience more therapeutic gain through an emotion-focused treatment (Ogrodniczuk et al., 2001; Matud, 2004; Ogrodniczuk, 2006; Meléndez et al., 2012) whereas men tend to react better to a problem-focused therapy which is more integrated in the CBT approach (Samoilov and Goldfried, 2000). More empirical evidence is required to investigate whether these findings can be replicated. We interpret these results with caution, taking into account the importance of the individual characteristics of the female participants in this considerably small sample size compared to the proportion of male participants.

The results presented here are highly explorative, and large prospective trials are needed to confirm or disprove the gender effects we demonstrate. It would be especially prudent for future studies to ensure an even inclusion of men and women, as the gender balance is disturbed in many studies, as well as in several of the therapy groups discussed in this paper. Furthermore, as we identified additional effects of symptoms of depression and hyperacusis, controlling for these factors is undeniably crucial.

We have demonstrated considerable effects of gender on tinnitus treatment outcome for different therapy options. Our results suggest that women might experience greater effects of orofacial physiotherapy and transcranial direct current stimulation compared to men. Within the psychotherapeutic treatments, we identified subtle gender differences between the outcome of CBT compared to eye movement desensitization and retraining, but mainly note that individual differences and psychiatric comorbidity affect the therapeutic pathway and treatment outcome. We report remarkable gender effects on treatment outcomes of several tinnitus therapy options. Overall, our results indicate that gender might be of influence for treatment outcome. Consequently, it might be important to consider gender when estimating the chance for treatment success.

\section{DATA AVAILABILITY STATEMENT}

The datasets generated for this study are available on request to the corresponding author.

\section{ETHICS STATEMENT}

The studies involving human participants were reviewed and approved by the Ethical committee of the University Hospital Antwerp. The patients/participants provided their written informed consent to participate in this study.

\section{AUTHOR CONTRIBUTIONS}

AV, TL, LJ, and NV contributed to data collection. AG and SM contributed to PI. VV, OV, VT, PV, and WD contributed to protocol and statistics. EC, SM, and AG contributed to analysis. $\mathrm{AG}, \mathrm{AV}$, TL, and EC contributed to manuscript writing. 


\section{REFERENCES}

Bowman, D., Scogin, F., Floyd, M., and McKendree-Smith, N. (2001). Psychotherapy length of stay and outcome: a meta-analysis of the effect of therapist sex. Psychotherapy 38, 142-148. doi: 10.1037/00333204.38.2.142

Budge, S. L., and Moradi, B. (2019). Gender identity. Psychother. Relationsh. Work 2:133.

Bueno, C. H., Pereira, D. D., Pattussi, M. P., Grossi, P. K., and Grossi, M. L. (2018). Gender differences in temporomandibular disorders in adult populational studies: a systematic review and meta-analysis. J. Oral Rehabil. 45, 720-729. doi: $10.1111 /$ joor. 12661

Cima, R. F., Andersson, G., Schmidt, C. J., and Henry, J. A. (2014). Cognitivebehavioral treatments for tinnitus: a review of the literature. J. Am. Acad. Audiol. 25, 29-61. doi: 10.3766/jaaa.25.1.4

Edvall, N. K., Gunan, E., Genitsaridi, E., Lazar, A., Mehraei, G., Billing, M., et al. (2019). Impact of temporomandibular joint complaints on tinnitus-related distress. Front. Neurosci. 13:879. doi: 10.3389/fnins.2019.00879

Felmingham, K. L., and Bryant, R. A. (2012). Gender differences in the maintenance of response to cognitive behavior therapy for posttraumatic stress disorder. J. Consult. Clin. Psychol. 80, 196-200. doi: $10.1037 / \mathrm{a} 0027156$

Frank, E., Schecklmann, M., Landgrebe, M., Burger, J., Kreuzer, P., Poeppl, T. B., et al. (2012). Treatment of chronic tinnitus with repeated sessions of prefrontal transcranial direct current stimulation: outcomes from an open-label pilot study. J. Neurol. 259, 327-333. doi: 10.1007/s00415-011-6189-4

Fumagalli, M., Vergari, M., Pasqualetti, P., Marceglia, S., Mameli, F., Ferrucci, R., et al. (2010). Brain switches utilitarian behavior: does gender make the difference? PLoS One 5:e8865. doi: 10.1371/journal.pone.0008865

Gilles, A., De Ridder, D., Van Hal, G., Wouters, K., Kleine Punte, A., and Van de Heyning, P. (2012). Prevalence of leisure noise-induced tinnitus and the attitude toward noise in university students. Otol. Neurotol. 33, 899-906. doi: 10.1097/MAO.0b013e31825d640a

Gilles, A., Schlee, W., Rabau, S., Wouters, K., Fransen, E., and Van de Heyning, P. (2016). Decreased speech-in-noise understanding in young adults with tinnitus. Front. Neurosci. 10:288. doi: 10.3389/fnins.2016.00288

Guideline Development Panel for the Treatment of PTSD in Adults, and American Psychological Association (2019). Summary of the clinical practice guideline for the treatment of posttraumatic stress disorder (PTSD) in adults. Am. Psychol. 74, 596-607. doi: 10.1037/amp0000473

Han, T. S., Jeong, J. E., Park, S. N., and Kim, J. J. (2019). Gender differences affecting psychiatric distress and tinnitus severity. Clin. Psychopharmacol. Neurosci. 17, 113-120. doi: 10.9758/cpn.2019.17.1.113

Henry, J. A., Dennis, K. C., and Schechter, M. A. (2005). General review of tinnitus: prevalence, mechanisms, effects, and management. J. Speech Lang Hear.Res. 48, 1204-1235. doi: 10.1044/1092-4388(2005/084)

Hiller, W., and Goebel, G. (2006). Factors influencing tinnitus loudness and annoyance. Arch. Otolaryngol. Head Neck Surg. 132, 1323-1330. doi: 10.1001/ archotol.132.12.1323

Jacquemin, L., Shekhawat, G. S., Van de Heyning, P., Mertens, G., Fransen, E., Van Rompaey, V., et al. (2018). Effects of electrical stimulation in tinnitus patients: conventional versus high-definition tDCS. Neurorehabil. Neural Repair 32, 714-723. doi: 10.1177/1545968318787916

Jastreboff, P. J. (2007). Tinnitus retraining therapy. Prog. Brain Res. 166, 415-423. doi: 10.1016/S0079-6123(07)66040-3

Jastreboff, P. J. (2015). 25 years of tinnitus retraining therapy. HNO 63, 307-311. doi: 10.1007/s00106-014-2979-1

Jastreboff, P. J., and Jastreboff, M. M. (2000). Tinnitus Retraining Therapy (TRT) as a method for treatment of tinnitus and hyperacusis patients. J. Am. Acad. Audiol. 11, 162-177.

Khalfa, S., Dubal, S., Veuillet, E., Perez-Diaz, F., Jouvent, R., and Collet, L. (2002). Psychometric normalization of a hyperacusis questionnaire. ORL J. Otorhinolaryngol. Relat. Spec. 64, 436-442. doi: 10.1159/000067570

Lefaucheur, J. P. (2016). A comprehensive database of published tDCS clinical trials (2005-2016). Neurophysiol. Clin. 46, 319-398. doi: 10.1016/j.neucli.2016.10.002

Lefaucheur, J. P., Antal, A., Ayache, S. S., Benninger, D. H., Brunelin, J., Cogiamanian, F., et al. (2017). Evidence-based guidelines on the therapeutic use of transcranial direct current stimulation (tDCS). Clin. Neurophysiol. 128, 56-92. doi: 10.1016/j.clinph.2016.10.087

Luders, E., Gaser, C., Narr, K. L., and Toga, A. W. (2009). Why sex matters: brain size independent differences in gray matter distributions between men and women. J. Neurosci. 29, 14265-14270. doi: 10.1523/JNEUROSCI.2261-09.2009

Lugo, A., Trpchevska, N., Liu, X., Biswas, R., Magnusson, C., Gallus, S., et al. (2019). Sex-specific association of tinnitus with suicide attempts. JAMA Otolaryngol. Head Neck Surg. 145, 685-687. doi: 10.1001/jamaoto.2019.0566

Luyten, T., Van de Heyning, P., Jacquemin, L., Van Looveren, N., Declau, F., Fransen, E., et al. (2019). The value of eye movement desensitization reprocessing in the treatment of tinnitus: study protocol for a randomized controlled trial. Trials 20:32. doi: 10.1186/s13063-018-3121-6

Matud, M. P. (2004). Gender differences in stress and coping styles. Pers. Individ. Diff. 37, 1401-1415. doi: 10.1016/j.paid.2004.01.010

Meeus, O. M., Spaepen, M., Ridder, D. D., and Heyning, P. H. (2010). Correlation between hyperacusis measurements in daily ENT practice. Int. J. Audiol. 49, 7-13. doi: 10.3109/14992020903160868

Meikle, M. B., Henry, J. A., Griest, S. E., Stewart, B. J., Abrams, H. B., McArdle, R., et al. (2012). The tinnitus functional index: development of a new clinical measure for chronic, intrusive tinnitus. Ear Hear. 33, 153-176. doi: 10.1097/ AUD.0b013e31822f67c0

Meléndez, J. C., Mayordomo, T., Sancho, P., and Tomás, J. M. (2012). Coping strategies: gender differences and development throughout life span. Span. J. Psychol. 15, 1089-1098. doi: 10.5209/rev_sjop.2012.v15.n3.39399

Meric, C., Gartner, M., Collet, L., and Chery-Croze, S. (1998). Psychopathological profile of tinnitus sufferers: evidence concerning the relationship between tinnitus features and impact on life. Audiol. Neurootol. 3, 240-252. doi: 10.1159/ 000013796

Michiels, S., De Hertogh, W., Truijen, S., and Van de Heyning, P. (2015a). Cervical spine dysfunctions in patients with chronic subjective tinnitus. Otol. Neurotol. 36, 741-745. doi: 10.1097/MAO.0000000000000670

Michiels, S., Van de Heyning, P., Truijen, S., and De Hertogh, W. (2015b). Diagnostic value of clinical cervical spine tests in patients with cervicogenic somatic tinnitus. Phys. Ther. 95, 1529-1535. doi: 10.2522/ptj.20140457

Michiels, S., De Hertogh, W., Truijen, S., and Van De Heyning, P. (2016a). The effect of cervical physical therapy in patients with cervicogenic somatic tinnitus. Man. Ther. 25:e102. doi: 10.1016/j.math.2016.05.180

Michiels, S., Naessens, S., Van de Heyning, P., Braem, M., Visscher, C. M., Gilles, A., et al. (2016b). The effect of physical therapy treatment in patients with subjective tinnitus: a systematic review. Front. Neurosci. 10:545. doi: 10.3389/ fnins.2016.00545

Michiels, S., Van de Heyning, P., Truijen, S., Hallemans, A., and De Hertogh, W. (2016c). Does multi-modal cervical physical therapy improve tinnitus in patients with cervicogenic somatic tinnitus? Man. Ther. 26, 125-131. doi: 10. 1016/j.math.2016.08.005

Michiels, S., Ganz Sanchez, T., Oron, Y., Gilles, A., Haider, H. F., Erlandsson, S., et al. (2018a). Diagnostic criteria for somatosensory tinnitus: a delphi process and face-to-face meeting to establish consensus. Trends Hear. 22:2331216518796403. doi: 10.1177/2331216518796403

Michiels, S., van der Wal, A. C., Nieste, E., Van de Heyning, P., Braem, M., Visscher, C., et al. (2018b). Conservative therapy for the treatment of patients with somatic tinnitus attributed to temporomandibular dysfunction: study protocol of a randomised controlled trial. Trials 19:554. doi: 10.1186/s13063-0182903-1

Michiels, S., Nieste, E., Van de Heyning, P., Braem, M., Visscher, C., Topsakal, V., et al. (2019). Does conservative temporomandibular therapy affect tinnitus complaints? A systematic review. J. Oral Facial Pain Headache 33, 308-317. doi: $10.11607 /$ ofph.2055

Michiels, S., Van de Heyning, P., Truijen, S., Hallemans, A., and De Hertogh, W. (2017). Prognostic indicators for decrease in tinnitus severity after cervical physical therapy in patients with cervicogenic somatic tinnitus. Musculoskelet. Sci. Pract. 29, 33-37. doi: 10.1016/j.msksp.2017.02.008

Ogrodniczuk, J. S. (2006). Men, women, and their outcome in psychotherapy. Psychother. Res. 16, 453-462. doi: 10.1080/10503300600590702

Ogrodniczuk, J. S., Piper, W. E., Joyce, A. S., and McCallum, M. (2001). Effect of patient gender on outcome in two forms of short-term individual psychotherapy. J. Psychother. Pract. Res. 10:69. 
Pattyn, T., Van Den Eede, F., Vanneste, S., Cassiers, L., Veltman, D. J., Van De Heyning, P., et al. (2016). Tinnitus and anxiety disorders: a review. Hear. Res. 333, 255-265. doi: 10.1016/j.heares.2015.08.014

Phillips, J. S., Erskine, S., Moore, T., Nunney, I., and Wright, C. (2019). Eye movement desensitization and reprocessing as a treatment for tinnitus. Laryngoscope 129, 2384-2390. doi: 10.1002/lary.27841

Pinto, P. C., Sanchez, T. G., and Tomita, S. (2010). The impact of gender, age and hearing loss on tinnitus severity. Braz. J. Otorhinolaryngol. 76, 18-24.

Rabau, S., Wouters, K., and Van de Heyning, P. (2014). Validation and translation of the Dutch tinnitus functional index. B ENT 10, 251-258.

Russell, M., Goodman, T., Wang, Q., Groshong, B., and Lyeth, B. G. (2014). Gender differences in current received during transcranial electrical stimulation. Front. Psychiatry 5:104. doi: 10.3389/fpsyt.2014.00104

Samoilov, A., and Goldfried, M. R. (2000). Role of emotion in cognitivebehavior therapy. Clin. Psychol. Sci. Pract. 7, 373-385. doi: 10.1093/ clipsy.7.4.373

Schlaepfer, T. E., Harris, G. J., Tien, A. Y., Peng, L., Lee, S., and Pearlson, G. D. (1995). Structural differences in the cerebral cortex of healthy female and male subjects: a magnetic resonance imaging study. Psychiatry Res. 61, 129-135. doi: 10.1016/0925-4927(95)02634-a

Seidman, M. D., and Jacobson, G. P. (1996). Update on tinnitus. Otolaryngol. Clin. North Am. 29, 455-465.

Seydel, C., Haupt, H., Olze, H., Szczepek, A. J., and Mazurek, B. (2013). Gender and chronic tinnitus: differences in tinnitus-related distress depend on age and duration of tinnitus. Ear Hear. 34, 661-672. doi: 10.1097/AUD. 0b013e31828149f2

Seydel, C., Haupt, H., Szczepek, A. J., Klapp, B. F., and Mazurek, B. (2010). Long-term improvement in tinnitus after modified tinnitus retraining therapy enhanced by a variety of psychological approaches. Audiol. Neurootol. 15, 69-80. doi: 10.1159/000231632

Swift, J. K., Callahan, J. L., Ivanovic, M., and Kominiak, N. (2013). Further examination of the psychotherapy preference effect: a meta-regression analysis. J. Psychother. Integr. 23:134. doi: 10.1037/a0031423
Tao, K. W., Owen, J., Pace, B. T., and Imel, Z. E. (2015). A meta-analysis of multicultural competencies and psychotherapy process and outcome. J. Counsel. Psychol. 62, 337-350. doi: 10.1037/cou0000086

Van de Heyning, P., Gilles, A., Rabau, S., and Van Rompaey, V. (2015). Subjective tinnitus assessment and treatment in clinical practice: the necessity of personalized medicine. Curr. Opin. Otolaryngol. Head. Neck. Surg 23, 369-375. doi: 10.1097/MOO.0000000000000183

Vielsmeier, V., Kleinjung, T., Strutz, J., Bürgers, R., Kreuzer, P. M., and Langguth, B. (2011). Tinnitus with temporomandibular joint disorders: a specific entity of tinnitus patients? Otolaryngol. Head Neck Surg. 145, 748-752. doi: 10.1177/ 0194599811413376

Villamar, M. F., Volz, M. S., Bikson, M., Datta, A., Dasilva, A. F., and Fregni, F. (2013). Technique and considerations in the use of $4 \times 1$ ring high-definition transcranial direct current stimulation (HD-tDCS). J. Vis. Exp. 77:e50309. doi: $10.3791 / 50309$

Zigmond, A. S., and Snaith, R. P. (1983). The hospital anxiety and depression scale. Acta Psychiatr. Scand. 67, 361-370.

Zoger, S., Svedlund, J., and Holgers, K. M. (2006). Relationship between tinnitus severity and psychiatric disorders. Psychosomatics 47, 282-288. doi: 10.1176/ appi.psy.47.4.282

Conflict of Interest: The authors declare that the research was conducted in the absence of any commercial or financial relationships that could be construed as a potential conflict of interest.

Copyright (C) 2020 Van der Wal, Luyten, Cardon, Jacquemin, Vanderveken, Topsakal, Van de Heyning, De Hertogh, Van Looveren, Van Rompaey, Michiels and Gilles. This is an open-access article distributed under the terms of the Creative Commons Attribution License (CC BY). The use, distribution or reproduction in other forums is permitted, provided the original author(s) and the copyright owner(s) are credited and that the original publication in this journal is cited, in accordance with accepted academic practice. No use, distribution or reproduction is permitted which does not comply with these terms. 